\title{
Optical vortex singularities and atomic circulation in evanescent waves
}

\author{
V. E. Lembessis ${ }^{\mathrm{a}}$, D. L. Andrews ${ }^{\mathrm{b}}, \mathrm{M}$. Babiker $^{\mathrm{c}}$ and S. Al-Awfid \\ ${ }^{a}$ New York College, 38 Amalias Avenue, GR 105 58, Athens, Greece \\ ${ }^{\mathrm{b}}$ School of Chemistry, University of East Anglia, Norwich NR4 7TJ, U.K. \\ ${ }^{c}$ Department of Physics, University of York, Heslington, York YO10 5DD, U.K. \\ ${ }^{d}$ Department of Physics, Taibah University, P.O. Box 30002, Medina, Saudi Arabia
}

The total internal reflection of an optical beam with a phase singularity can generate evanescent light that displays a rotational character. At a metalized surface, in particular, field components extending into the vacuum region possess vortex properties in addition to surface plasmon features. These surface plasmonic vortices retain the phase singularity of the input light, also mapping its associated orbital angular momentum. In addition to a two-dimensional patterning on the surface, the strongly localized intensity distribution decays with distance perpendicular to the film surface. The detailed characteristics of these surface optical vortex structures depend on the incident beam parameters and the dielectric mismatch of the media. The static interference of the resulting surface vortices, achieved by using beams suitably configured to restrict lateral in-plane motion, can be shown to give rise to optical forces that produce interesting dynamical effects on atoms or small molecules trapped in the vicinity of the surface. As well as trapping within the surface plasmonic fields, model calculations reveal that the corresponding atomic trajectories will typically exhibit a variety of rotational and vibrational effects, significantly depending on the extent and sign of detuning from resonance.

Keywords: singular optics, surface plasmons; optical vortices, optical manipulation; atoms at surfaces, atom lithography, optical tweezers, optical spanner

\section{INTRODUCTION}

When laser light is strongly coupled to material excitations, it can produce a variety of hybrid 'dressed photon' or polariton modes. A major new area of photonics research has blossomed from exploitation of the very distinctive hybrid excitations known as surface plasmons, which arise when laser light emerges in vacuum at an interface with an electronically dense and delocalized medium. ${ }^{[1,2]}$ These hybrid optical/material excitations propagate along the planar surface, but their field amplitudes decay exponentially with distance normal to the surface, and they have an upper limit of frequency dependent upon the electronic density of the material. Surface plasmons are now very well characterized, and their dispersion relations are sufficiently reproducible that they can be used for calibration purposes. Although most surface plasmon studies involve laser beams of conventional beam structure, there has been considerable recent interest in the effects produced by using beams with a helical wavefront structure - twisted beams or optical vortices. The propensity of such beams to convey orbital angular momentum permits the generation of surface plasmon optical vortices (SPOVs), i.e. optical vortices with surface plasmon polariton features. The electromagnetic fields so created can be unusually strong, and so will couple strongly to matter localized in the vicinity of the surface.

Surface plasmon optical vortices attract attention for a variety of reasons and potential applications. There are interesting links with circular polarization effects; for example Ohdaira et al ${ }^{[3]}$ have shown that the superposition of two crosspropagating evanescent waves on a planar dielectric surface can generate circularly polarized propagating waves in free space, as a result of near-field interactions with a probe of sub-wavelength dimensions. As a counterpart to this effect, Gorodetski et al. ${ }^{[4]}$ have established that spin-based plasmonic effects can be created in nanostructures by the use of circularly polarized light, exploiting a geometric phase associated with a locally variant polarization state. In related developments Kim et al. ${ }^{[5]}$ have recently shown how the fabrication and deployment of a plasmonic vortex lens can be used to generate plasmon vortices and dynamically switch their topological charge, whilst $\mathrm{Hu}$ et al ${ }^{[6]}$ have proposed a method for focusing surface plasmons on a flat metal film by the use of radially polarized beam which shares the axial singularity feature associated with optical vortices.

Complex Light and Optical Forces V, edited by David L. Andrews, Enrique J. Galvez, Jesper Glückstad, Proc. of SPIE Vol. 7950, 795007 · @ 2011 SPIE · CCC code: 0277-786X/11/\$18 · doi: 10.1117/12.874926 
The aim of the present study is to identify behavior associated with the optical angular momentum of surface plasmon optical vortices, whose origin is neither photon spin nor surface morphology. Particular attention focuses on the dynamical characteristics of an atom close to the surface, in response to a SPOV. In this connection it is notable that a form of surface plasmon can be excited on the surface of a metallic film, of finite thickness, deposited on a planar dielectric substrate. The evanescent character of this type of surface mode is manifest in the exponential decay of its electromagnetic fields with distance from the surface, in the vacuum region. Such an excitation persists as a well defined entity even when the metallic film is very thin, with dielectric properties that can in practice be represented as those of a two-dimensional metallic sheet. Full details of the underlying theory, which can only be sketched here, have been given in earlier reports. ${ }^{[7,8]}$

\section{THE STRUCTURE OF VORTEX EVANESCENT FIELDS}

Consider the case of a single vortex beam with a Laguerre-Gaussian (LG) beam profile, impinging from within a dielectric support at an angle if incidence $\phi$, on a metallic film of thickness $d$. The assumption is that, before the LG light is switched on, one or more atoms are trapped vertically by an optical trap. The depth of the well is assumed to be greater than $k_{\mathrm{B}} T$, and significantly stronger than the potential created by the evanescent light - the latter decaying exponentially in the $z$-direction. This is a typical Kretschmann configuration, widely used for surface plasmon studies, as illustrated in Fig. 1 (a) below.

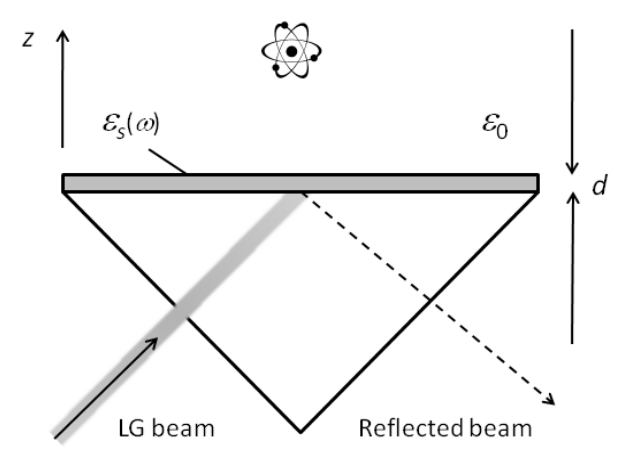

(a)



(b)

Fig. 1. Schematic representation of an atom in a vacuum, trapped above the point on a surface where there is total internal reflection of a Laguerre-Gaussian beam; the dielectric interface is embossed with a metallic film, supporting surface plasmon vortices: $(a)$ single-beam geometry; $(b)$ counterpropagating beam geometry.

The SPOV fields are made subject to boundary and phase-matching conditions and the evanescent field in the vacuum region is expressible in cylindrical coordinates in the form;

$$
\boldsymbol{E}_{k l p}(x, y, z>0)=\frac{2 A_{k l p}(x, y)}{\zeta}\left(\hat{\boldsymbol{x}}+i \frac{k_{\|}}{\mathrm{k}_{\mathrm{z} 1}} \hat{\boldsymbol{z}}\right) e^{-i\left(x n \frac{\omega}{c} \sin \phi+k_{z 2} d\right)} e^{-k_{z 1} z}
$$

where carets denote unit vectors, $\omega$ is the frequency of the light, $n$ the refractive index of the dielectric, and

$$
\zeta=\left(1-i \frac{k_{z 2} \varepsilon_{1}}{k_{z 1} \varepsilon_{2}}\right) \cosh \left(k_{z s} d\right)+\left(\frac{k_{z s} \varepsilon_{1}}{k_{z 1} \varepsilon_{s}}-i \frac{k_{z 2} \varepsilon_{s}}{k_{z s} \varepsilon_{2}}\right) \sinh \left(k_{z s} d\right) .
$$

For a Laguerre-Gaussian mode of order $l$ and degree $p$, the explicit form of the factor $A$ is: 


$$
\begin{aligned}
A_{k \mid p}(x, y) & =\frac{\xi_{k 00} N_{\ell p}}{\left(2^{p+\ell \mid \ell} p ! \ell ! !\right)^{1 / 2}}\left[\frac{\sqrt{2\left(y^{2}+(x \cos \phi-d \sin \phi)^{2}\right)}}{w_{0}}\right]^{|\ell|} \exp \left[\frac{-\left(y^{2}+(x \cos \phi-d \sin \phi)^{2}\right)}{w_{0}^{2}}\right] L_{p}^{\mid \ell}\left[\frac{\left(y^{2}+(x \cos \phi-d \sin \phi)^{2}\right)}{w_{0}^{2}}\right] \\
& \times \exp \left[-i \ell \arctan \left(\frac{y}{x \cos \phi-d \sin \phi}\right)\right]
\end{aligned}
$$

Here, $\boldsymbol{k}_{\square}$ is the in-plane wave-vector and $k_{z 1}, k_{z 2}, k_{z 5}$ are propagation wave-vectors in regions where the dielectric functions are respectively $\varepsilon_{1}, \varepsilon_{2}, \varepsilon_{\mathrm{s}}$, with region 1 taken as vacuum and region 2 the dielectric of refractive index $n$, while the metallic film has a frequency-dependent dielectric function in the Drude form, $\varepsilon_{s}=1-\omega_{p}^{2} / \omega^{2}$, with $\omega_{p}$ the plasma frequency.

\section{ATOMIC MOTION (SINGLE BEAM CASE)}

We now introduce an atom in the vacuum region at position $\boldsymbol{R}(\mathrm{t})=(x(t), y(t), z(t)), z>0$. For simplicity its electronic properties are cast in the simple form of a two-level system, with level separation $\omega_{0}$ and width $\hbar \Gamma_{0}$. The interaction of the atom with the surface plasmon vortex is taken in the dipole approximation, with a Hamiltonian $H_{\text {int }}=-\boldsymbol{\mu} \cdot \boldsymbol{E}_{k l p}(\boldsymbol{R})$. The interaction of the atom with the electric field of the plasmonic surface vortex leads to two important dynamical attributes of the motion, namely the Rabi frequency $\Omega_{k l p}(\boldsymbol{R}(t))$ and phase $\Theta\left(\boldsymbol{R}_{\square}(t)\right)$, each parametrically dependent on time; these emerge as follows;

$$
\begin{gathered}
\Omega_{k \mid p}(\boldsymbol{R}(t))=\frac{2 \mu\left(1+k_{\|}^{2} / k_{z 1}^{2}\right)^{1 / 2}}{\hbar|\zeta|} \frac{\xi_{k 00} N_{\ell p}}{\left(2^{p+|l|} p ! \mid \ell !\right)^{1 / 2}}\left[\frac{\sqrt{2\left(y^{2}+(x \cos \phi-d \sin \phi)^{2}\right)}}{w_{0}}\right]^{|\ell|} L_{p}^{|l|}\left(\frac{\left(y^{2}+(x \cos \phi-d \sin \phi)^{2}\right)}{w_{0}^{2}}\right) e^{-k_{z 1}{ }^{2}} e^{-\left(y^{2}+(x \cos \phi-d \sin \phi)^{2}\right) / w_{0}^{2}} \\
\Theta_{l}(x(t), y(t))=\left[\ell \arctan \left(\frac{y}{x \cos \phi-d \sin \phi}\right)\right]-\left(\frac{n x \omega \sin \phi}{c}\right) ;
\end{gathered}
$$

the latter phase is a function only of the in-plane variables. The steady-state optical force acting on the atom in the vacuum region, when its velocity vector is $\boldsymbol{V}(t)=\dot{\boldsymbol{R}}(t)$, is the sum of a dissipative force and a dipole force, each of which is dependent on both position and velocity;

$$
\boldsymbol{F}_{\text {total }}(\boldsymbol{R}, \boldsymbol{V})=\boldsymbol{F}_{\text {diss }}(\boldsymbol{R}, \boldsymbol{V})+\boldsymbol{F}_{\text {dip }}(\boldsymbol{R}, \boldsymbol{V})=2 \hbar\left\{\frac{\Gamma_{0} \Omega^{2}(\boldsymbol{R}) \nabla \Theta-2 \hbar \Delta(\boldsymbol{R}, \boldsymbol{V}) \Omega(\boldsymbol{R}) \nabla \Omega(\boldsymbol{R})}{\Delta^{2}(\boldsymbol{R}, \boldsymbol{V})+2 \Omega^{2}(\boldsymbol{R})+\Gamma_{0}^{2}}\right\}
$$

where the mode labels are dropped for convenience, and $\Delta_{0}=\omega-\omega_{0}$ is the detuning of the light from the atomic transition frequency. The equation of motion of the atom is given by Newton's Second Law, and its solution requires specifying the position and velocity of the atom at $t=0$, when the beam is switched on. The solutions are straightforward.

An atom engulfed in an SPOV will be subject to optical forces tending to produce linear propulsion along the surface, as well as forces that can trap it within the localized evanescent fields. To obviate longitudinal motion necessarily associated with the lack of symmetry in the present configuration, it is more expedient to introduce a second LG beam one that has the same mode attributes (including intensity) except for one crucial feature; its topological charge is chosen to have the same magnitude but opposite sign to the other beam. The second beam thus enters the system along the path of reflection for the original beam, as shown in Fig. $1(b)$. In consequence there will be a cancellation of unidirectional translational atomic motion, whereas the torques associated with the two beams add to give a resultant that is cast about the normal to the surface, the $z$-direction. This enables the atomic motion to be confined to a path in the surface plane. 


\section{ATOMIC MOTION RESULTING FROM COUNTERPROPAGATING LG BEAMS}

In the twin-beam configuration, the dynamics again follows a Newtonian equation of motion, driven by a sum of the dissipative and dipole forces delivered by each beam. To secure results for the dynamical motion, the RK4 Runge-Kutta method has been used to numerically solve by iterative convergence the resulting differential equations of atomic motion. To illustrate the solutions leading to typical trajectories we consider the dynamics of a sodium atom in a surface plasmon, on a thin silver film deposited on glass. The plasmon is formed using two confocal, counter-propagating 'doughnut' Laguerre-Gaussian modes and with parameter values as follows: wavelength $\lambda=589 \mathrm{~nm}$; beam-waist $w_{0}=$ $35 \lambda ; \varepsilon_{1}=1 ; \varepsilon_{2}=2.298 ; \Gamma_{0}=6.13 \times 10^{7} \mathrm{~Hz} ; d=59 \mathrm{~nm} ; \mu=2.6 e a_{0}$ ( $e$ is the electron charge, $a_{0}$ the Bohr radius); $\left|\Delta_{0}\right|=10^{2} \Gamma_{0}$. The atom is initially at rest at a position $\left(x_{0}, y_{0}\right)=\left(0.5 w_{0}, 0.5 w_{0}\right)$. The calculations show that positive detuning engenders an outwardly spiraling orbital motion leading the atom away from the beam axis. However in the case of negative detuning the trajectory of the sodium atom is very different, as is immediately apparent from the typical trajectories shown in Fig. 2.



(a)
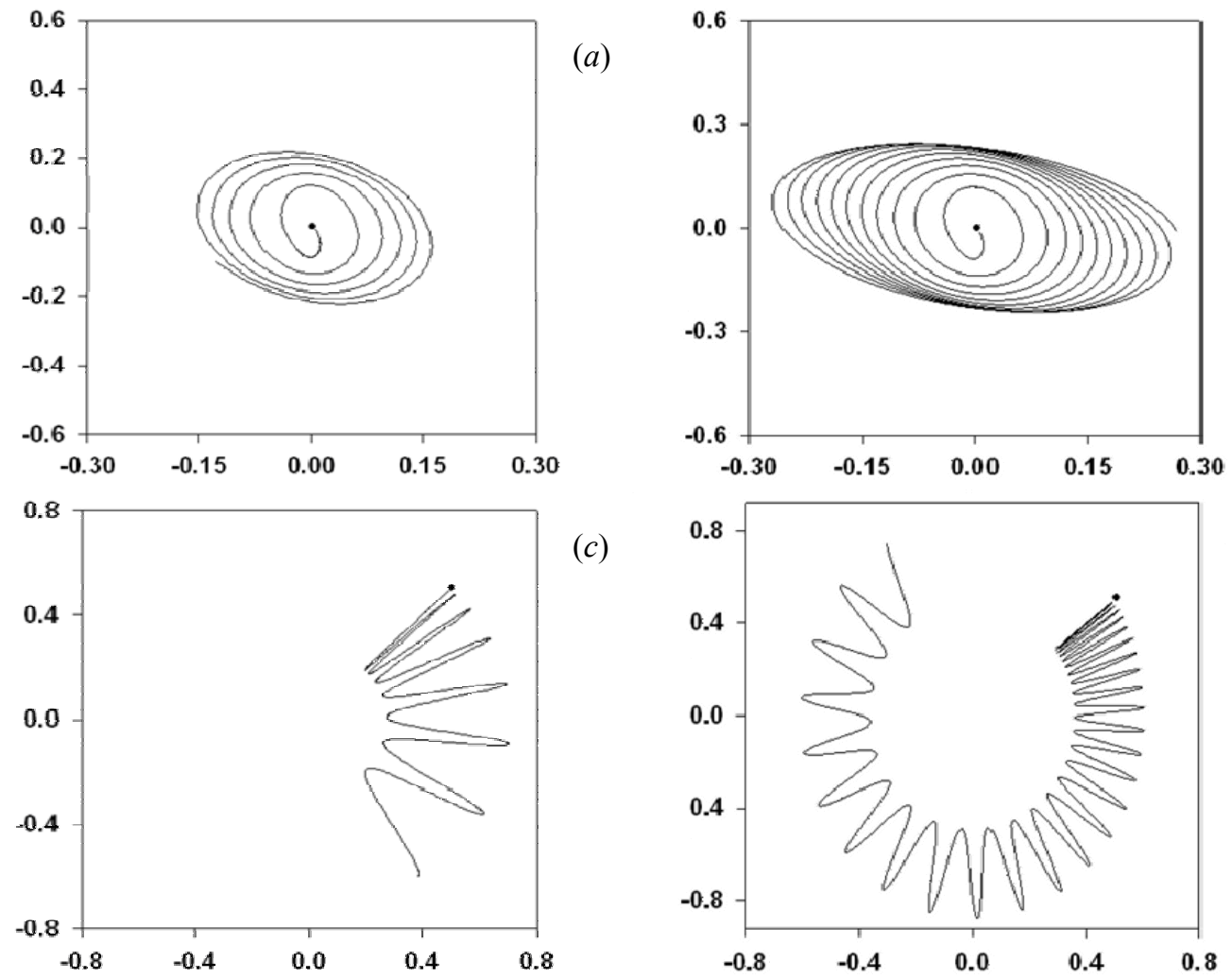

(c)



$(d)$

Fig. 2. Atomic trajectories (vertical axis, $y / w_{0}$ where $w_{0}$ is the beam-waist; horizontal axis $x / w_{0}$ : $(a)$ positive detuning, $\ell_{1}=-\ell_{2}=1, p=0 ; \phi_{1}=-\phi_{2}=\pi / 4$, input beam irradiance $I=2 \times 10^{4} \mathrm{~W} \mathrm{~m}^{-2} ;(b)$ positive detuning, $\ell_{1}=-\ell_{2}=2, p=0 ;$ $\phi_{1}=-\phi_{2}=\pi / 4, I=2 \times 10^{9} \mathrm{~W} \mathrm{~m}^{-2} ;(c)$ negative detuning, $\ell_{1}=-\ell_{2}=1, p=0 ; \phi_{1}=-\phi_{2}=\pi / 4, I=2 \times 10^{4} \mathrm{~W} \mathrm{\textrm {m } ^ { - 2 }} ;(d)$ negative detuning, $\ell_{1}=-\ell_{2}=2, p=0 ; \phi_{1}=-\phi_{2}=\pi / 4, I=2 \times 10^{8} \mathrm{~W} \mathrm{~m}^{-2}$.

All of the trajectories manifest an obvious circulation due to the resultant torque, the only common feature in all of the presented results. An outwardly spiraling trajectory, such as shown in Fig. $2(a)$ and $(b)$, characterizes an incompletely bound form of potential that arises in the case of positive detuning, whereas when the detuning is negative, a radial vibrational force operates within a bound potential to which the atom is subject. In the latter case there is now a confinement region in the form of an elliptical concentric valley, defined by the intensity distribution of the $l=1, p=0$ 
LG mode; radial confinement of the atom leads to a vibrational motion in a radial direction, resulting in an overall zigzag trajectory. Acceleration of the orbiting motion is also apparent. The spiraling out in the positive-detuning case and the accelerating zigzag in the negative detuning case both arise from the light-induced torque associated with the orbital angular momentum of the evanescent light.

\section{DISCUSSION}

The nature and implications of the results reported here add a new dimension to the possibilities for atomic manipulation using structured light. ${ }^{[9]}$ Based on the optical torque that was first predicted ${ }^{[10,11]}$ for two-level atoms, some more recent theory work has shown that microscale ion current loops, atomic rings and cubic clusters can be generated using an LG beam-induced optical molasses configuration. ${ }^{[12]}$ In the broader context of atomic motions produced by LaguerreGaussian light, it is interesting to reflect on the pioneering work of Andersen et al., ${ }^{[13]}$ which established a new protocol for an almost frictionless circulation of ultracold sodium atoms, also using counter-propagating LG beams to create the torque. More recently two of us (VEL, MB) have shown that it is possible for a persistent current flow to be produced in an atomic gas Bose-Einstein condensate, using counterpropagating LG beams to create a toroidal trap. ${ }^{[14]}$ The theory, based on a two-photon process within three atomic levels, leads to a quantized light-induced torque that rotates the atoms, generating an atomic current flow in the ring. The optical input not only creates a torque influencing rotational motion; it also leads to axial cooling of the atomic motion. In such a system it proves possible for the torque to be switched and controlled by varying the frequencies of the incident light, thereby allowing a mechanism for the control of the current flow.

The main work discussed in the present paper serves to exemplify the physics associated with vortex characteristics in an evanescent electromagnetic field generated in vacuum, outside the metalized surface of an optically dense dielectric medium. To illustrate the mechanical effects of the associated torque, we have considered the trajectory of a model sodium atom in the evanescent field, governed by a surface plasmon optical vortex generated by the simplest LG vortex, the $(1,0)$ mode. The initial positioning of the atom has been marginally offset from the central common focus of the two input beams, where there is a phase singularity - although it is known that quantum uncertainty principles allow even an atom at the center of such a vortex to interact with local fields. ${ }^{[15,16]}$ The dynamics of the atom in the SPOV fields thus produced by two symmetrically disposed and internally reflected, counter-propagating, LG modes has been evaluated, and shown to allow two very different types of in-plane trajectories, governed by the detuning of the light from the closest atomic transition frequency.

This is a theory that in principle applies to SPOVs generated by LG light modes with any degree of topological charge indeed to any optical mode characterized by an optical vortex. Amongst the possible applications, one can envisage the generation of carefully designed SPOV arrays being used to create a pre-determined two-dimensional pattern for the manipulation and the surface deposition of atoms, a technique that has possible relevance to the fields of nanolithography and other methods that involve the motion of atomic components localized near a surface. The theory is also readily extendable to systems in which the evanescent mode couples into a fluid, rather than vacuum. For example, it is conceivable that the rotary motions of larger particles, trapped by optical tweezers near a fluid surface, might support the optomechanical production of hydrodynamic vortices - a new form of optical spanner action. In the context of rapid ongoing developments in the fields of microfluidics and 'lab-on-a-chip' devices, ${ }^{[17,18]}$ the opportunities that might be afforded by such advances are indeed enticing.

\section{REFERENCES}

[1] Raether, H., [Surface Plasmons on Smooth and Rough Surfaces and on Gratings], Springer Verlag, Berlin (1988).

[2] Zayats, A. V., Smolyaninov, I. I. and Maradudin, A. A., "Nano-optics of surface plasmon polaritons," Phys. Rpts 408, 131-314 (2005). 
[3] Ohdaira, Y., Inoue, T., Hori, H. and Kitahara, K., "Local circular polarization observed in surface vortices of optical near-fields," Opt. Express 16, 2915-2921 (2008).

[4] Gorodetski, Y., Niv, A., Kleiner, V. and Hasman, E., "Observation of the Spin-Based Plasmonic Effect in Nanoscale Structures,” Phys. Rev. Lett. 101, 043903 (2008).

[5] Kim. H., Park, J., Cho, S.-W., Lee, S.-Y., Kang, M. and Lee, B., "Synthesis and dynamic switching of surface plasmon vortices with plasmonic vortex lens," Nano Lett. 10, 529-536 (2010).

[6] Hu, Z. J., Tan, P. S., Zhu, S. W. and Yuan, X.-C., "Structured light for focusing surface plasmon polaritons," Opt. Express 18, 10864-10870 (2010).

[7] Lembessis, V. E., Babiker, M. and Andrews, D. L., "Surface optical vortices," Phys. Rev. A 79, 011806(R) (2009)

[8] Andrews, D. L., Babiker, M., Lembessis V. E. and Al-Awfi, S., "Surface plasmons with phase singularities and their effects on matter," Phys. Status Solidi RRL 4, 241-243 (2010).

[9] Babiker, M. and Andrews, D. L., "Atomic and molecular manipulation using structured light," [Structured Light and Its Applications: An Introduction to Phase-Structured Beams and Nanoscale Optical Forces], ed. D.L. Andrews, pp. 169-194, Academic, Burlington MA (2008).

[10] Babiker, M., Power, W. L. and Allen, L., "Light-induced torque on moving atoms," Phys. Rev. Lett. 73, 1239-1242 (1994).

[11] Power, W. L., Allen, L., Babiker, M. and Lembessis, V. E., "Atomic motion in light beams possessing orbital angular momentum," Phys. Rev. A 52, 479-488 (1995).

[12] Carter, A. R., Babiker, M., Al-Amri, M. and Andrews, D. L., "Generation of microscale current loops, atom rings and cubic clusters using twisted optical molasses," Phys. Rev. A 73, 021401(R) (2006).

[13] Andersen, M. F., Ryu, C., Claude, P., Natarajan, V., Vaziri, A., Helmerson, K. and Phillips, W. D., “Quantized Rotation of Atoms From Photons with Orbital Angular Momentum,” Phys. Rev. Lett. 97, 170406 (2006).

[14] Lembessis, V. E. and Babiker, M., "Light-induced torque for the generation of persistent current flow in atomic gas Bose-Einstein condensates," Phys. Rev. A 82, 051402(R) (2010).

[15] Berry, M. V. and Dennis, M. R., "Quantum cores of optical phase singularities," J. Opt. A: Pure Appl. Opt. 6, S178-S180 (2004).

[16] Barnett, S. M., “On the quantum core of an optical vortex,” J. Mod. Opt. 55, 2279-2292 (2008).

[17] Glückstad, J., “Microfluidics: sorting particles with light,” Nature Materials 3, 9-10 (2004).

[18] Edel, J. B. and deMello, A. J., [Nanofluidics. Nanoscience and Nanotechnology], Royal Society of Chemistry Publishing, Cambridge (2009). 